\title{
Empirical Modeling of Public Safety Voice Traffic in the Land Mobile Radio Band
}

\author{
Tanim M. Taher, Roger B. Bacchus, Kenneth J. Zdunek \\ Department of Electrical and Computer Engineering \\ Illinois Institute of Technology, Chicago, IL
}

\author{
Dennis A. Roberson, \\ Department of Computer Science \\ Illinois Institute of Technology, Chicago, IL
}

\begin{abstract}
An RF measurement system with high time resolution is implemented to determine the statistical characteristics of various channels in the Land Mobile Radio bands. The applicability of simple statistical models to the observed data is investigated, as well as their validity over short and long periods of time. The results show that the statistics of the idle and holding times of communication on these channels vary significantly over time and demonstrate daily periodicity, requiring non-stationary models to accurately represent them. Over short durations of time however, conventional distributions such as the exponential and log-normal may adequately characterize the properties of these quantities, allowing convenient and compact representations of the data. Results based on empirical data are presented to quantify the probability of stationarity for voice traffic within a time span of given length. The findings are useful for network planning or streamlining, network simulation and modeling, and investigation of dynamic spectrum access.

Index Terms-cognitive radio; dynamic spectrum access; voice traffic; land mobile radio; public safety radio
\end{abstract}

\section{INTRODUCTION}

The Land Mobile Radio (LMR) channels have been allocated by the FCC primarily for voice communications by state and local government agencies, and commercial entities [KOB01]. Public safety agencies like police and fire departments use LMR systems for communication between dispatch centers and mobile field agents, or for direct mobile-to-mobile communications. Similarly, commercial users often employ LMR for "walkie-talkie" mode two-way communications. In urban areas, most of the limited number of LMR channels available in the VHF (148-174 MHz) and UHF (450-512 $\mathrm{MHz}$ ) bands are already allocated to specific users. At the same time it has been observed that most of the time this allocated spectrum is not fully utilized [BAC08, BAC10]. Hence, the LMR bands are candidates for studying the feasibility of Dynamic Spectrum Access (DSA) technology to improve the capacity utilization in these highly sought after frequencies. Particularly, during emergency situations when federal, state and city agencies may converge in a geographic locale [FCC08, FCC09], DSA could be applied to increase the pool of voice channels available for public safety use [TAH11].

The characterization of radio activity is an important step in the design of a communication system [ASC09], particularly for cognitive radios that must transparently coexist alongside legacy implementations and make optimal use of limited spectrum. This activity is often random, and effective characterization requires the use of statistical models that may be derived from some fundamental knowledge of the nature of the activity, or from empirical data. The former is often impractical except for simple, idealized cases, while the latter offers greater flexibility, at the significant expense of requiring previously measured traffic data. For traffic characterization purposes, empirical data of radio usage in the $450 \mathrm{MHz} \mathrm{LMR}$ band was extracted from RF measurements conducted at the Illinois Institute of Technology (IIT) in Chicago.

In this paper we investigate modeling the distribution and time variations of channel characteristics that include the interarrival, holding and idles times of various radio channels with simple and analytically tractable representations. Previous studies [BAR97, ASC07] have extensively examined these very characteristics for public safety users, but generally over shorter observation periods. We extend this research by using empirical data collected over weeks and for multiple public safety LMR channels. We also explore the periodicity and non-stationary aspects of the traffic characteristics.

This paper is organized as follows. Section II gives an overview of the measurement system. Section III examines histograms of channel holding, idle and inter-arrival times over long time periods; Section IV compares these results with short-term histograms and discusses the effects of nonstationarity. Applications, conclusions and future work are presented in Section V.

\section{Measurement OVERVIEW}

A low-cost high speed RF data acquisition and storage system was implemented to measure voice channels in the $450 \mathrm{MHz}$ LMR band, as shown in Figure 1. The system utilizes a USRP N200 software radio platform and a WBX frontend [ETT12] (tunable between $50 \mathrm{MHz}$ and $2.2 \mathrm{GHz}$ ) to obtain time-domain samples. These are then used to estimate the Power Spectrum via Fast Fourier Transform (FFT), from which the power within every $12.5 \mathrm{kHz}$ channel span is found by integration. These power values are then stored in a database for later analysis or for real-time web-streaming. Considerable effort went into the robust design and optimization of this database system, both in terms of hardware and software; especially, since about 1 gigabyte of data is collected daily.

The system was installed on the roof of the 22 story IIT Tower approximately $5 \mathrm{~km}$ south of the central Chicago business district, and conveniently across the street from the 


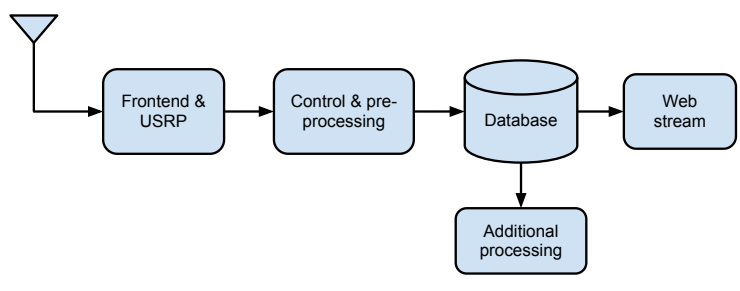

Figure 1. System block diagram

Headquarters of the Chicago Police Department (CPD). The system includes an omni-directional $50 \mathrm{MHz}-1.3 \mathrm{GHz}$ discone antenna. A 450-474 MHz bandpass filter and a fixed $10 \mathrm{~dB}$ attenuator were added to reduce out of band interference and distortion of the WBX frontend. The measurement parameters were also adjusted to reduce spurious artifacts in the received signal.

The system is capable of measuring the power in each $12.5 \mathrm{kHz}$ channel with a time resolution of $250 \mathrm{~ms}$, sufficient enough to allow the observation of public safety and commercial voice traffic. Lab testing of a commercially available LMR radio (Motorola Radius CP200 [MOT12]) indicated a minimum transmission duration of $500 \mathrm{~ms}$. Hence, a sampling time of $250 \mathrm{~ms}$ is adequately small to capture most, if not all events in these channels. Every 10 minutes, the measurement system also stores the high frequency resolution $(\sim 50 \mathrm{~Hz})$ FFT based plots of the max-hold and average power spectrum within that 10 minute window. Figure 2 illustrates a sample max-hold plot, which is useful in post-processing for classifying the signals; for example, an analog FM has a very different spectrum signature from digital P25 LMR [PRO12].

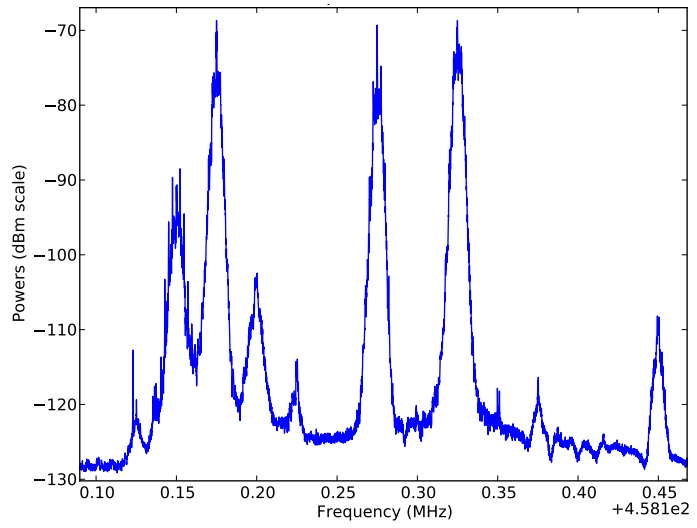

Figure 2. Max-hold spectral plot showing multiple LMR channels

For this paper, we analyzed data collected over a five week period starting from September 15th, 2011 in the 453-465 $\mathrm{MHz}$ frequency range. The date range was selected as there were no maintenance related periods of interruption in the measurement system and the data is continuous over the five weeks.

\section{LONG-TERM CHANNEL STATISTICS}

CPD uses 21, $25 \mathrm{kHz}$ bandwidth analog FM broadcast channels with center frequencies uniformly spaced at 25 $\mathrm{kHz}$ intervals starting from 460.025 to $460.525 \mathrm{MHz}$. The measurement system has a frequency resolution of $12.5 \mathrm{kHz}$ and therefore measures two frequency points per channel every $250 \mathrm{~ms}$. Statistics for the "idle time" durations, "hold times", and "inter-arrival times" were all developed through analysis of this data.

The thresholds to determine activity were determined experimentally for each LMR channel by examining histograms of the channel powers. In most cases the transmit power was high enough to create a clear distinction between activity and non-activity; hence the error in classifying the two should be low.

Idle time is defined as the time period between transmissions, when there is no detectable signal power in the LMR channel. No attempt is made to group a series of calls in to conversations. Hence, all the longer idle times separating conversations and all the shorter gaps between the calls within a conversation are treated together for analysis in this paper. Hold time is the duration of transmissions, that is, the period over which the channel has power that exceeds a threshold. Finally, inter-arrival time [BAR99] is the time duration between the start of one transmission and the next, and is equivalent to the sum of two, consecutive hold and idle times.

\section{A. Idle Times}

Figure 3 shows a histogram of the idle time durations for the CPD channel centered at $460.125 \mathrm{MHz}$, measured between $12.01 \mathrm{am}$, September 15th to $11.59 \mathrm{pm}$, September 21st, 2011 (seven days). Also shown are several model distributions that were used for curve-fitting this histogram.

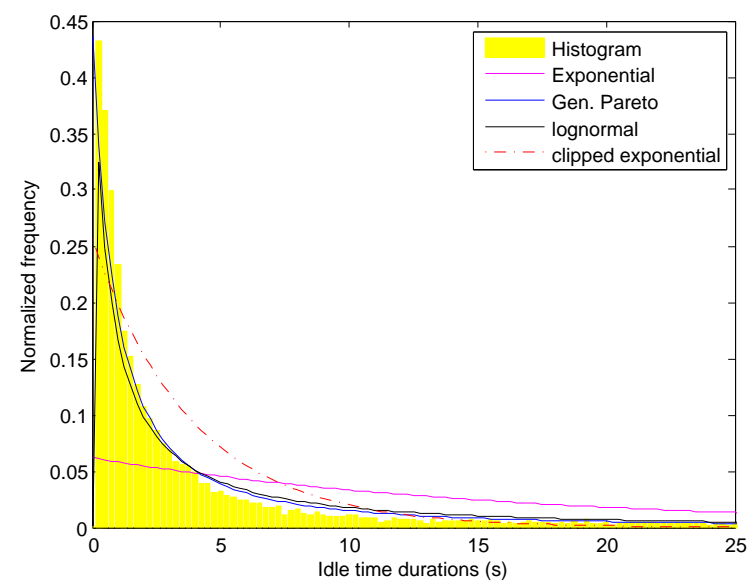

Figure 3. Histogram of idle times with model fits (week long data)

The general shape of the histogram is reminiscent of the exponential distribution - which is commonly used to model idle times [SHA04]. However, the histogram is heavy-tailed and does not decay very fast. Consequently the arrival rate for 
a standard exponential model to fit the data gives a skewed result that does not match the idle time distribution very closely. An alternative "clipped" exponential model was tried, where only the idle time durations between 0 and 30 seconds were used to estimate the arrival-rate parameter. This model performed better, but of course fails to take account of the heavy tail idle times greater than 30 seconds. For this CPD channel, $82 \%$ of the idle durations fell within the 30 second clipping time, and $18 \%$ were heavy tail data.

The generalized Pareto distribution performed reasonably for shorter idle time durations. However, this distribution decays slowly and the fit gives a higher probability for long idle times than what was actually observed. The best fit for the long term idle time data collected over the one week was obtained with a log-normal distribution.

To quantify the fit, the mean squared error (MSE) between the histogram and the estimated distribution for each type of model was calculated. The average MSE across the 21 channels for each model was calculated and normalized with the largest value of the error. This is shown in Table 1.

Table I

NORMALIZED MSE BETWEEN HISTOGRAM OF IDLE TIMES AND MODEL FITS

\begin{tabular}{|c|c|c|}
\hline Distribution & MSE (1 week duration) & MSE (2 hour duration) \\
\hline \hline Exponential & 1.000 & 1.000 \\
\hline Generalized Pareto & 0.917 & 0.960 \\
\hline Log-normal & 0.409 & 0.555 \\
\hline Clipped exponential & 0.611 & 0.524 \\
\hline
\end{tabular}

Of the simple distributions considered, the log-normal distribution most closely fits the measurements, followed by the clipped exponential model. The standard exponential model fits poorly with the histogram and has a MSE two and a half times higher than the log-normal distribution. Interestingly, previous papers have discussed the modeling of the idle time as a memory-less exponentially distributed random variable [HES81, HOW10]. Graphically, the generalized Pareto distribution seems to follow the histogram well, but the MSE is quite high.

\section{B. Hold Times}

A histogram showing the distribution of the channel hold times is shown in Figure 4, along with several candidate distributions (exponential, generalized Pareto, log-normal and gamma). The clipped exponential model was not considered as the hold time data is not heavy-tailed. The data shown in this plot is taken from the same one week time period as the idle times data.

Unlike the idle time, the holding time distribution possesses a distinctly non-zero peak at approximately 2.25 seconds a reasonable value for typical push to talk voice communications. The exponential and Pareto distributions show poor fits; however, the log-normal and gamma distributions show a good representation. The MSE across the 21 CPD channels normalized to the worse case fit (Pareto in this case) is shown in Table 2.

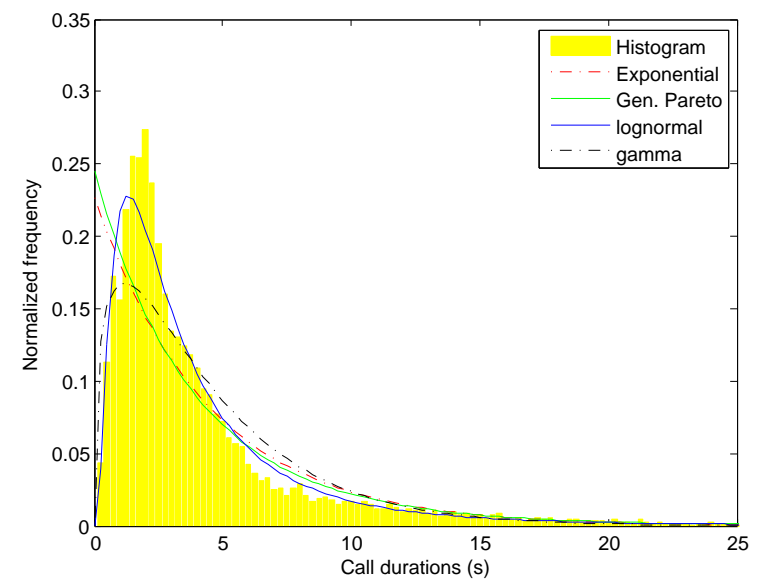

Figure 4. Histogram of hold times with model fits (week long data)

Table II

NORMALIZED MSE BETWEEN HISTOGRAM OF HOLD TIMES AND MODEL FITS

\begin{tabular}{|c|c|c|}
\hline Distribution & MSE (1 week duration) & MSE (2 hour duration) \\
\hline \hline Exponential & 0.855 & 0.951 \\
\hline Generalized Pareto & 1.000 & 1.000 \\
\hline Log-normal & 0.180 & 0.324 \\
\hline Gamma & 0.274 & 0.524 \\
\hline
\end{tabular}

The models discussed are valid for the majority of channels observed which possess uni-modal distributions; however several cases were observed where the holding time was substantially different. Figure 5 shows the distribution of the holding times taken from several different channels in the same LMR band over a one day period.

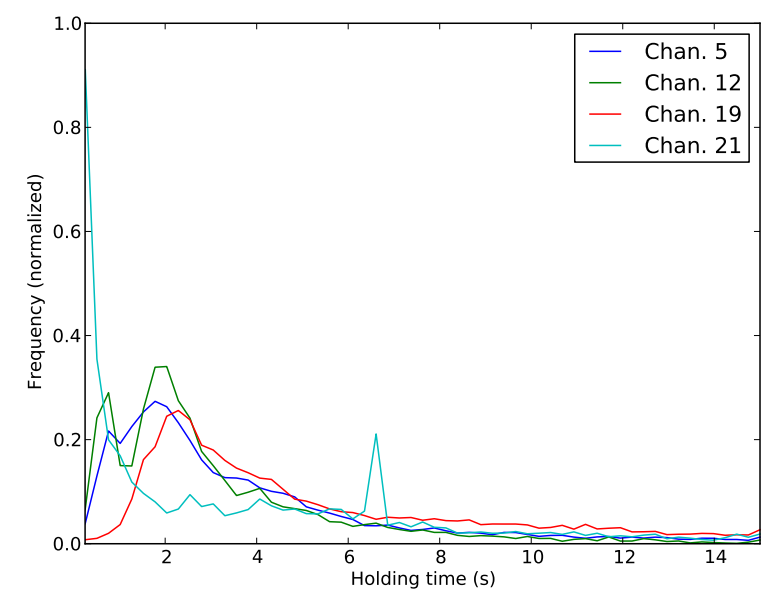

Figure 5. Histograms of hold times, illustrating atypical channels

Most channels follow the typical log-normal shape (e.g. channels 5, 19), but others (12 and 21) are more exponentially distributed, or possess peaks at multiple locations. The channels demonstrating such behavior may be used for non-voice applications or just have atypical usage. Network simulation studies of public safety [ASC07] LMR would 
require special models for treatment of such less common channels; alternatively, the actual traffic data measured and stored in the database (see Section II) is usable to source traffic information in the simulations.

\section{Inter-arrival Times}

A histogram of the inter-arrival times along with various candidate distributions is shown in Figure 6, for the same dataset as previously discussed.

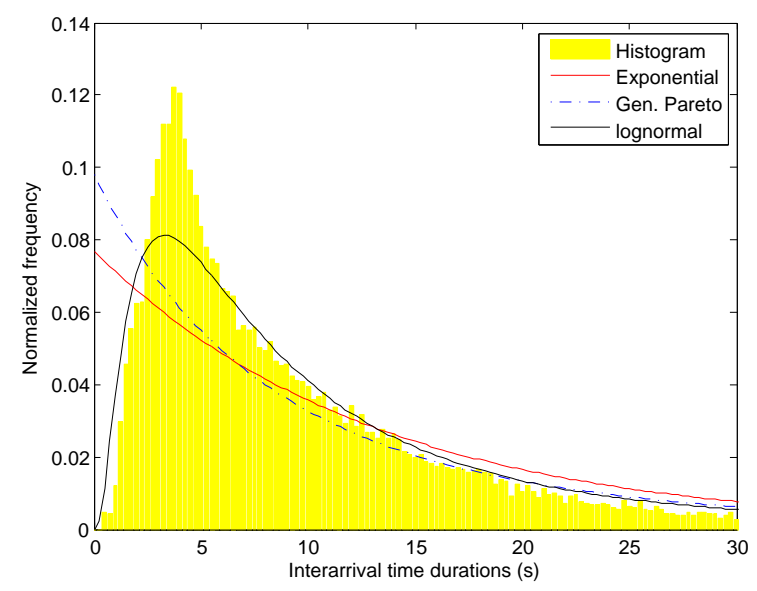

Figure 6. Histogram of inter-arrival times with model fits (week long data)

The inter-arrival time is generated from the sum of the holding and idle times. In this case, the shape of the histogram is dominated by that of the holding time, possessing a peak at around $3.75 \mathrm{~s}$. However, the distribution is not as well approximated by the log-normal distribution, as demonstrated by the MSE values in Table 3. Some more accurate models consist of a mixture of log-normal distributions [SAN98].

Table III

NORMALIZED MSE BETWEEN HISTOGRAM OF INTER-ARRIVAL TIMES AND MODEL FITS

\begin{tabular}{|c|c|c|}
\hline Distribution & MSE (1 week duration) & MSE (2 hour duration) \\
\hline \hline Exponential & 0.990 & 0.988 \\
\hline Generalized Pareto & 1.000 & 1.000 \\
\hline Log-normal & 0.728 & 0.830 \\
\hline
\end{tabular}

\section{Time Series of PARAMEters for Short-TERM MODELING}

The histograms and models previously discussed were based on data collected over a period of days. It is likely that statistics such as the holding, idle and inter-arrival times will be non-stationary and possess periodicity and other trends. The histograms for the idle times, hold times, and inter-arrival times were recalculated for a two hour duration at lunch hour on a weekday. The standard distributions introduced in Section III were again used to model the histograms. As an example, Figure 7 shows the histogram of idle times and the model fits for one CPD channel. There are gaps in the histogram as the total number of calls within the two hours is lower compared to the seven day period in Section III. Due to these gaps (zero values), the cumulative errors between the histogram and the model distributions are higher for the short term study (higher MSE).

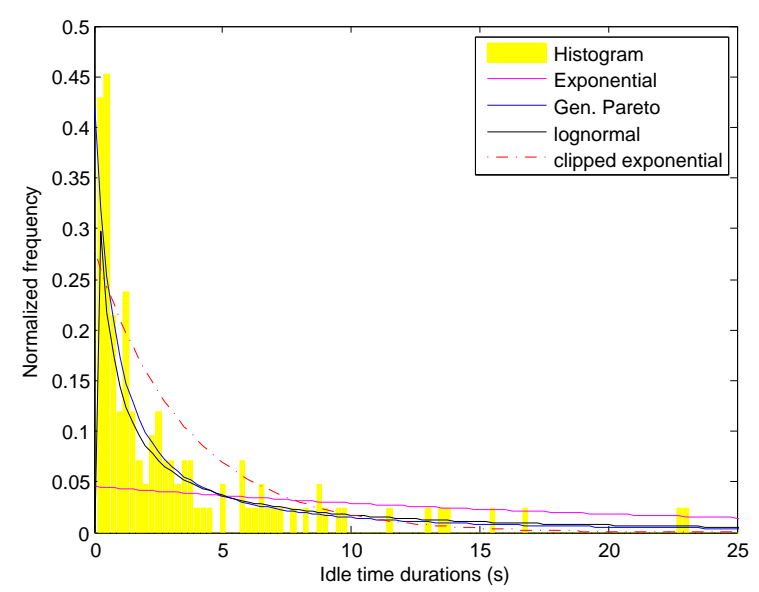

Figure 7. Histogram of idle times with model fits (two hour long data)

The MSE values for each of the three traffic characteristics were calculated for all $21 \mathrm{CPD}$ channels, and the mean MSE values for the idle, hold and inter-arrival times across the 21 channels were normalized. The results are presented in Tables 1, 2 and 3 in the third columns labeled as "MSE (2 hour duration)". For the short term idle time durations, the clipped exponential has the least MSE. It is observed that for modeling long term idle time data a log-normal distribution performs best, but over shorter periods, the clipped exponential fits slightly better than the log-normal. For the hold and interarrival times, the log-normal distribution was found to have the closest fit both for short and long terms. The major difference observed between the long term and short term modeling was related to the estimated values of the parameters for the models, indicating that these parameters are not stationary over longer periods of time.

The plot in Figure 8 shows a time-series plot of the mean idle and holding times within 30 minute windows. The data was collected over a 7 day period beginning on 15/9/2011 (a Thursday) and was smoothed with a three hour moving average filter. Daily weekday and weekend trends are evident in both the idle and holding times; the hold times showing peaks during the daytime, and lower levels during the weekend. Similar periodicity was seen in the time series of the statistical parameters for the inter-arrival times.

This non-stationary behavior implies that the previous models in Section III are not appropriate over shorter periods of time as the activity is modulated by the date and time of day. One option to extend the applicability of these results is to model the relevant distribution parameters as a time series, creating a dynamic model which can be updated at periodic intervals. For example, the reciprocal of the mean hold times plotted in Figure 8 are estimates of the rate parameter if a simple, but less accurate, exponential model is used to model 


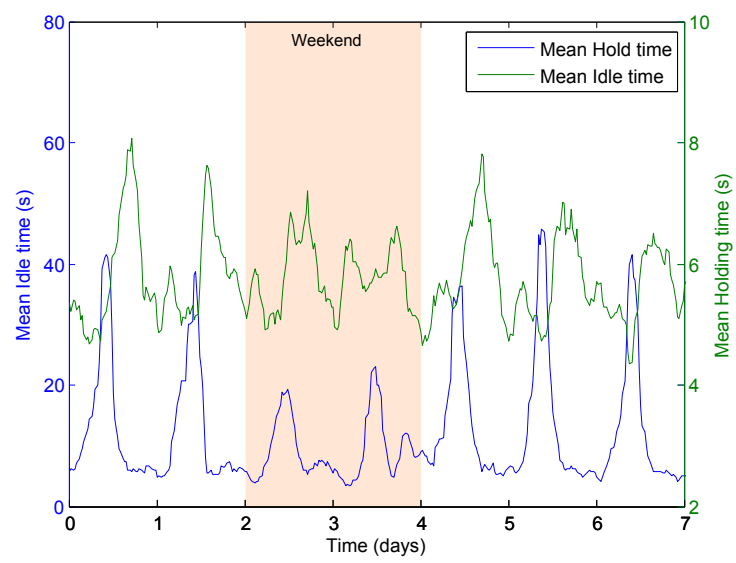

Figure 8. Time series of hold and idle time statistics for a CPD channel

the voice call durations.

As another example, Figure 9 shows the variation of the two parameters for the log-normal distribution model of idle times. The parameters were calculated for every 30 minute window during a one week period, and each time series was smoothed with a 3 hour moving average filter. The two time series' are again periodic, and show significant differences from the log-normal distribution's parameter values estimated from the long-term data (represented by the two horizontal lines in Figure 9).

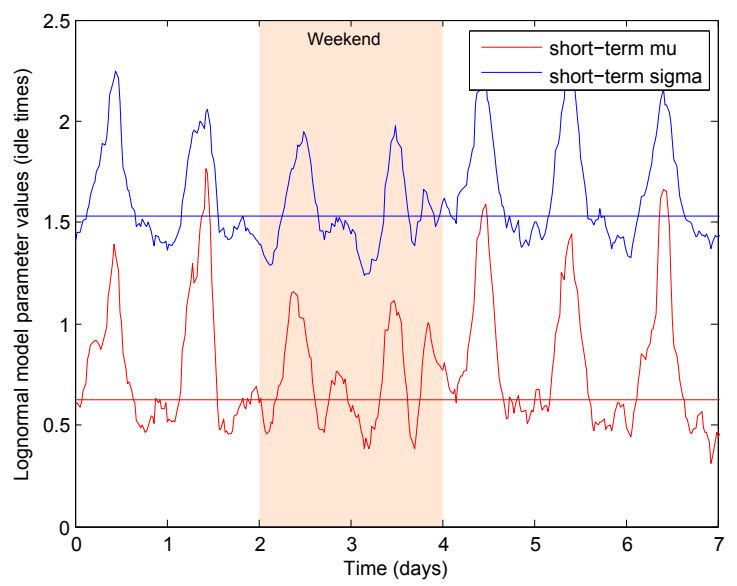

Figure 9. Time series of log-normal parameter variations for modeling idle time characteristics

For simulation studies, a simple model that captures the variation of the statistical parameters is useful to dynamically track the statistics of the voice traffic data. Dynamic spectrum access technologies may make it possible for multiple voice traffic sources to share the same RF channel. As part of ongoing research, we are investigating the feasibility of channel sharing and collision probabilities if two or more public safety channels shared the same RF frequency, or if a public safety user opportunistically shares the frequency with a low duty cycle commercial user [TAH11]. In such simulations, the statistical parameters for the traffic sources have to be periodically updated, where the update period should be short enough such that the model parameters remain stationary.

A method was devised to investigate the limitations of simple stationary models of idle and holding time statistics over long periods of time, utilizing the Kwiatkowski, Phillips, Schmidt and Shin (KPSS) test for stationarity [KWI92]. For each of the 21 CPD channels, the voice activity over 35 days was divided into bins of a fixed time length. The KPSS test with a 5\% significance level was then applied to the data in each bin. The percentage of bins that passed the test was recorded. This analysis was then repeated for bin sizes starting from 6 minutes to 600 minutes. The results are plotted in Figure 10 for both idle and hold times.

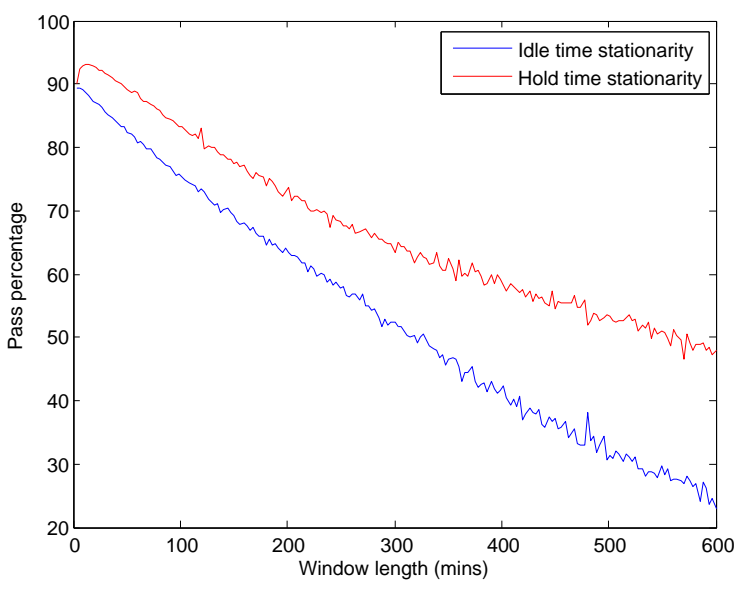

Figure 10. Fraction of bins passing KPSS test vs. window length for 21 CPD channels (five weeks of data)

The results show that a 30 minute window for the hold time is $91 \%$ likely to be stationary, and $79 \%$ for a 2 hour window. For simulation studies, for example, this suggests the parameter updates should be done at frequent intervals $(<30$ minutes) to ensure the model is applicable.

Figures 8-10 illustrates the importance of considering stationarity when examining voice traffic for long periods of time. One approach to incorporate this behavior is to represent the parameters of the distribution over short, successive windows (where a stationary assumption is more valid) by a seasonal auto-regressive integrated moving average (SARIMA) time series model [CHA84] that captures its periodicities, correlations and other dynamics. A trivial example for the $\sigma$ parameter of the log-normal model that would be applicable during weekdays (where the day-to-day periodicity is consistent and any short term correlation is neglected) could be: $\sigma_{t+24}=\sigma_{t}$.

A potential problem with using such SARIMA models is that there is no constraint to limit the quantity being represented to physically realizable values. For an exponential model, for example, the rate parameter should strictly be nonnegative, however there will be a non-zero probability of this condition being violated if it is modeled using a simple SARIMA model. One common approach to address this is to 
model the exponential of this parameter [LEW76].

\section{CONClusions AND Future WORK}

The results of this paper show that the commonly used log-normal distribution is a good model for characterizing both channel holding and idle times in public safety LMR radio channels, over various time periods. The inter-arrival time statistics are more difficult to quantify with simple distributions. As may be expected, the probability of the traffic statistics within a window being stationary decreases with the time length of the window. This was demonstrated quantitatively using a large set of 21 channels and empirical data obtained over five weeks. To account for the inherent nonstationarity in the statistics, each parameter of these simple models can be characterized as a time series that incorporates dynamic behavior and periodicities.

As part of ongoing research, we are studying the feasibility of a cognitive radio that can opportunistically use the LMR channels in emergencies. Such a cognitive radio needs channel characteristics to rank the channels that it can opportunistically use. Once an unoccupied channel has been selected for secondary transmission, both the primary user's (commercial user) idle time and the secondary user's (DSA public safety radio) hold time statistics become important to predict how long the secondary user can continue to stay in the channel with minimal interference probability. A plot (e.g. Figure 10) showing the probability of stationarity versus window length is useful as it gives quantitative guidelines on how often the channels' statistics must be updated for the cognitive radio to perform optimally.

In [TAH11], a comparison was made between the traffic volume of emergency responders and commercial radio users during an emergency situation caused by a blizzard. The measurement system used [TAH11] was not capable of getting the high time resolution data necessary to create statistical distribution models of the public safety radio traffic during that emergency, as was done for this paper using a new high-speed LMR measurement system. Since the new current system is capturing data continuously, in the future we expect to capture some emergency events and observe how the statistics of traffic distribution for public safety radio users change during such times. This future work should help to better illustrate the potential of DSA technology in the LMR band to improve capacity for emergency responders .

The statistical studies of public safety voice channels will be extended to include commercial and business LMR channels. In our cognitive radio study, the business users will be the primary transmitters, and the results that have been presented for public safety LMR will be used to model the secondary opportunistic access user.

\section{ACKNOWLEDGMENT}

The authors would like to acknowledge the National Science Foundation (NSF). This research is supported by the NSF under Grant No. 1018786. The authors are grateful to Jeffrey Engel and Philip Felber for their extensive contributions to the design and implementation of the RF measurement system and the database described in Section II.

\section{REFERENCES}

[ASC07] Aschenbruck, N.; Martini, P.; Gerharz, M., "Characterisation and Modelling of Voice Traffic in First Responder Networks," Proc. IEEE Conf. on Local Computer Networks (LCN2007), 2007

[ASC09] Aschenbruck, N.; Martini, P., "Modeling public safety scenarios to evaluate wireless communication systems," Wireless Communication, Vehicular Technology, Information Theory and Aerospace \& Electronic Systems Technology, 2009. Wireless VITAE 2009. 1st International Conference on, vol., no., pp.510-514, 17-20 May 2009

[BAC08] Bacchus, R.B.; Fertner, A.J.; Hood, C.S.; Roberson, D.A., "LongTerm, Wide-Band Spectral Monitoring in Support of Dynamic Spectrum Access Networks at the IIT Spectrum Observatory," New Frontiers in Dynamic Spectrum Access Networks, 2008. DySPAN 2008. 3rd IEEE Symposium on, vol., no., pp.1-10, 14-17 Oct. 2008

[BAC10] Bacchus, R.B.; Taher, A.J.; Zdunek, K.J.; Roberson, D.A., "Spectrum Utilization Study in Support of Dynamic Spectrum Access for Public Safety," New Frontiers in Dynamic Spectrum Access Networks, DySPAN 2010. 4th IEEE Symposium, Singapore, April. 2010

[BAR97] Barcelo, F.; Bueno, S., "Idle and inter-arrival time statistics in public access mobile radio (PAMR) systems," Global Telecommunications Conference, 1997. GLOBECOM '97., IEEE , vol.1, no., pp.126-130 vol.1, 3-8, Nov 1997

[BAR99] Barcelo, F; Sánchez, J.I., "Probability distribution of the InterArrival time to Cellular Telephony Channels", IEEE 49th Vehicular Technology Conference (VTC Spring), pp. 762-766., 1999

[CHA84] Chatfield, C., The Analysis of Time Series: An Introduction; Chapman and Hall, 3rd edition; 1984

[ETT12] Ettus Research - Products, Retrieved January 30, 2012 from https://www.ettus.com/product

[FCC08] FCC Public Safety and Homeland Security Bureau; "Emergency Communications during the Minneapolis Bridge Disaster," Technical Case Study; Nov. 2008

[FCC09] FCC Public Safety and Homeland Security Bureau; "Emergency Communications During Hurricane Ike," Technical Case Study, Dec. 2009

[HES81] Hess G.; Cohn J., "Communications load and delay in mobile trunked systems," Proc. IEEE Vehicular Technology Conf., Washington, DC, Apr. 1981, pp. 269-273

[HOW10] Lee, H.; Cho D., "Capacity Improvement and Analysis of VoIP Service in a Cognitive Radio System," Vehicular Technology, IEEE Transactions on, vol.59, no.4, pp.1646-1651, May 2010

[KOB01] Kobb. B; Wireless Spectrum Finder: Telecommunications, Government and Scientific Radio Frequency Allocations in the US $30 \mathrm{MHz}$ - $300 \mathrm{GHz}$, McGraw Hill, 2001

[KWI92] Kwiatkowski, D.; Phillips, P. C. B.; Schmidt, P.; Shin, Y., "Testing the Null Hypothesis of Stationarity against the Alternative of a Unit Root." Journal of Econometrics. Vol. 54, 1992, pp. 159-178

[LEW76] Lewis, P.A.; Shedler, G.S., "Statistical Analysis of Non-stationary Series of Events in a Data Base System". IBM Journal on Research and Development, 20:465-482, 1976

[MOT12] Motorola Radius CP200 Portable, Retrieved January 30, 2012 from http://www.2wayradios.com/cp200.htm

[PRO12] Project 25 group, Retrieved January 30, 2012 from http://www.project25.org/

[SAN98] Sánchez, J.I.; Barceló, F., "Inter-arrival Time Distribution for Channel Arrivals in Cellular Telephony", Proc. 5th International Workshop on Mobile Multimedia Communication, pp. 245-254, Berlin 1998

[SHA04] Sharp, D.S.; Cackov, N.; Laskovic, N.; Qing Shao; Trajkovic, L., "Analysis of public safety traffic on trunked land mobile radio systems," Selected Areas in Communications, IEEE Journal on, vol.22, no.7, pp. 1197- 1205, Sept. 2004

[TAH11] Taher, T.M.; Bacchus, R.B.; Zdunek, K.J.; Roberson, D.A.; "Dynamic spectrum access opportunities for public safety in land mobile radio bands," Cognitive Radio Oriented Wireless Networks and Communications (CROWNCOM), 2011 Sixth International ICST Conference on , vol., no., pp.355-359, 1-3 June 2011 